\title{
Influence of smoking cessation drugs on blood pressure and heart rate in patients with cardiovascular disease or high risk score: real life setting
}

André Pacheco Silva ${ }^{1}$, Jaqueline Scholz ${ }^{2 *}$, Tania Ogawa Abe ${ }^{2}$, Gabriela Gouveia Pinheiro ${ }^{1}$, Patricia Viviane Gaya ${ }^{1}$, Alexandre Costa Pereira ${ }^{3}$ and Paulo Caleb Junior Lima Santos ${ }^{3}$

\begin{abstract}
Background: Smoking is the most important reversible cardiovascular risk factor. It is well established that quitting smoking reduces coronary events. However, on several occasions, the cardiovascular safety of smoking cessation drugs has been questioned. Our goal is to evaluate the effects of smoking cessation drugs on blood pressure and heart rate in patients from a smoking cessation service in a cardiology hospital.

Methods: We examined the PAF database (Smoking Cessation Assistance Program database) between January 2008 and March 2014. We analyzed data from 900 patients who were compliant with the treatment (50.5\% male, average age $53 \pm 17$ years). The most frequent clinical diagnoses were coronary artery disease (25.2\%), hypertension $(57.2 \%)$, and diabetes (13.4\%). Blood pressure, heart rate, and carbon monoxide (CO) concentration in exhaled air were analyzed at consecutive visits during the first 45 days of treatment (mean visits - 3). Analysis of repeated measures was used for the statistical analysis $(p<0.05)$.

Results: Two hundred seventy one patients used nicotine replacement therapy (NRT) alone, 81 used bupropion alone, 154 used varenicline alone, 283 used NRT plus bupropion and 111 used bupropion plus varenicline. For all smoking cessation drugs, used alone or in combination, no increase occurred in the average value of systolic blood pressure (SBP), diastolic blood pressure (DBP) and heart rate (HR). Significant reductions in CO concentrations occurred in all smoking cessation drug groups.

Conclusion: Smoking cessation drugs used in monotherapy or in combined regimens did not influence systolic blood pressure (SBP), diastolic blood pressure (DBP) and heart rate (HR) in this group of patients during the observation period.
\end{abstract}

Keywords: Smoking cessation, Nicotine replacement therapy, Bupropion, Varenicline, Cardiovascular disease, Hypertension

\section{Background}

Smoking cessation substantially lowers the risk of death in patients of all ages [1], including those with cardiovascular disease [2]. The long-term cardiovascular benefits of smoking cessation are well established $[1,2]$. Clinical practice guidelines [3] recommend the use of cessation

\footnotetext{
* Correspondence: jaquelineissa@yahoo.com.br

${ }^{2}$ Smoking Cessation Program Department, Heart Institute (InCor), University of Sao Paulo Medical School, Av. Dr. Eneas de Carvalho Aguiar 44, Cerqueira Cesar, 05403-900 Sao Paulo, SP, Brazil

Full list of author information is available at the end of the article
}

pharmacotherapy for smokers interested in quitting. The first-line drugs for smoking cessation are nicotine replacement therapy (NRT - gum or patch), bupropion (Zyban, Wellbutrin) and varenicline (Chantix, Champix).

These medications demonstrated effectiveness in achieving complete smoking cessation [3], but their safety in patients with cardiovascular $(\mathrm{CV})$ disease has been questioned and evaluated in several studies and metaanalyses. Previous reports associated NRT use with occurrence of cardiovascular events, such as myocardial infarction, especially in patients who continued smoking 
[4]. However, two recent meta-analyses showed an increase in CV symptoms, including tachycardia and nonspecific chest pain $[5,6]$ but not in MACEs - defined as cardiovascular death, nonfatal myocardial infarction and nonfatal stroke [6]. With bupropion, trials including smokers with cardiovascular disease did not report a statistically significant increase in $\mathrm{CV}$ events compared to placebo. However, the sample sizes were small and not powered for safety [7-9].

A recent network meta-analysis of 21 randomized clinical trials (RCTs) showed that bupropion was not harmful for MACEs and appeared to have a cardioprotective effect [6]. Varenicline, an alpha-4 beta- 2 nicotinic acetylcholine receptor partial agonist, binds to the same receptors as nicotine, which is known to have sympathomimetic cardiovascular effects [10]. The first meta-analysis on the CV safety of varenicline, predominantly composed of trials excluding patients with $\mathrm{CV}$ disease, concluded that increased risk (72 \%) exists for minor and major cardiovascular events among tobacco users [11]. A more recent large meta-analysis with more than 9,000 patients, however, found no significant risk of major CV events associated with varenicline use $[6,12]$. Trials that included patients with a history of CV disease found that even in a higher risk population, varenicline did not add significant damage $[6,12]$.

There are few studies evaluating combination of therapies for smoking cessation [13-15], none of them evaluating safety in patients with cardiovascular disease. One of these studies excluded patients with cardiovascular diseases or history of hypertension [15]. In the other studies there were not reports of major or minor adverse cardiovascular events during the smoking cessation therapy $[13,14]$. There is not meta-analysis available in the literature until this moment.

We examined CV effects of these drugs on blood pressure and heart rate in outpatient setting according to different combination therapies and the previous presence of hypertension, coronary artery disease, and/or acute myocardial infarction.

\section{Methods}

We analyzed 900 outpatient subjects followed at the Smoking Cessation Program of the Heart Institute (InCor), University of Sao Paulo Medical School, Sao Paulo, Brazil. Most of the patients have cardiovascular diseases or high risk score. We used the PAF database (Smokers Assistance Program) between January 2008 and March 2014 and looked up subjects of both genders older than 18 years of age. The Ethics Committee for Research Project Evaluation (CAPPesq) of the Hospital das Clinicas School of Medicine, University of Sao Paulo, approved the study. The study complied with the Declaration of Helsinki. They agreed with the informed consent form.
During the study period, 1791 patients were enrolled to the service. However, 891 patients were excluded because they did not come in the consecutive visits and their data for BP, HR, and/or $\mathrm{CO}$ were not available. However, patients with analyzed data $(n=900)$ did not have significant differences in their SBP, DBP, HR and $\mathrm{CO}$ values $(125 \pm 21 \mathrm{mmHg} ; 76 \pm 12 \mathrm{mmHg} ; 74 \pm 13 \mathrm{bpm}$; $11 \pm 10 \mathrm{ppm})$ compared with data of the untreated patients $(n=891)(123 \pm 22 \mathrm{mmHg} ; 74 \pm 18 \mathrm{mmHg} ; 74 \pm$ 18 bpm; $12 \pm 12$ ppm $)(p>0.05)$.

Thus, 900 patients were included in this analysis. They received smoking cessation medication to be taken for at least 12 weeks. Among the available medications, there were nicotine replacement therapies (NRT - patch and gum), bupropion, and varenicline. In the first visit (baseline), the patient was not in use of any drug for smoking cessation. One of these drugs was prescribed as monotherapy according to the nicotine dependence level, previous use of smoking cessation drugs, availability of the medication and lack of absolute contraindication related to each drug. Varenicline was prescribed for patients who failed in previous attempts with NRT and/or bupropion, or who smoked one or more packs of cigarettes per day.

The gap between follow-up visits was 2 to 3 weeks. In all visits the patient were questioned about smoking status, compliance and collateral effects of the medication prescribed, and we evaluated the vital signs, CO level, and withdrawal symptoms.

In the second visit, the patients who achieved success with monotherapy and had no or mild withdrawal symptoms were kept with the same medication. The patients who did not achieve success and the patients who achieved complete cessation but with moderate or intense withdrawal symptoms had associated therapies [13].

The data were categorized in 3 visits in sequential follow-up, called initial (baseline), second, and third visits. Variables from the database included in the analysis were: age; sex; smoking cessation; smoking cessation drugs used; smoker's degree of nicotine dependence according to the Fagerström score [16] and Issa score [17] at the initial visit; presence or absence of hypertension, diabetes mellitus type 2 (DMT2) and coronary artery disease (CAD); acute myocardial infarction (AMI); obesity, heart failure, arrhythmias, or valvular heart disease; and the number of cardiovascular medications being used at the initial visit. All the visits and measurements were done during the afternoon period. In each visit, the median of 2 blood pressures was taken with a mercury sphygmomanometer, and heart rate and exhaled $\mathrm{CO}$ level were collected. The parameter we used for smoking cessation was CO level below 6 ppm [18] associated with the self-reported cessation. The data regarding systolic blood pressure (SBP), diastolic blood 
pressure (DBP), and heart rate (HR) of study patients were compared for initial, second, and third visits, according to smoking cessation drugs used, alone or in combination, as well as between 2 groups: first with the presence of hypertension, CAD, and/or AMI and second without all these diseases.

\section{Statistical analysis}

Categorical variables are presented as percentages and continuous variables are presented as means \pm SD (standard deviation). Friedman's test (repeated measures) was performed to analyze SBP, DBP, HR, and CO levels during visits, according to the prescribed pharmacotherapy and the presence of hypertension, CAD, or AMI. Friedman's test was also performed to analyze SBP, DBP, and HR according with the CO cutoff $(6 \mathrm{ppm})$ in the second and third visits and the presence of hypertension, CAD, or AMI. Statistical analyses were carried out using SPSS 16.0 software (IBM, New York, NY), with the level of significance set at $p<0.05$.

\section{Results}

During the study, 900 patients were included in the analysis. The median age of patients was $53 \pm 17$ and $50.5 \%$ were male. Table 1 shows demographic and clinical characteristics. Smoker's degree of nicotine dependence was $6.3 \pm 2.3$ assessed by Fagerström score [16] and $2.9 \pm 0.9$ by Issa score [17]. From the 900 patients included, 580 (64.4 \%) had hypertension, CAD, and/or AMI. Hypertension was the most common cardiovascular disease (57.2 \%), and a significant number of patients had coronary artery disease $(25.2 \%)$ and previous acute myocardial infarction $(27.0 \%)$.

Among these 900 patients, 271 patients used NRT alone, 81 used bupropion alone, 154 used varenicline alone, 283 used NRT + bupropion, and 111 used bupropion + varenicline.

Table 2 shows the sequential measures of blood pressures, heart rate and $\mathrm{CO}$ concentration of patients according to prescribed drugs and the presence of hypertension,

Table 1 Demographic and clinical characteristics of study patients

\begin{tabular}{ll}
\hline Variables & $n=900$ \\
\hline Sex, female (\%) & 49.5 \\
Fagerström score & $6.3 \pm 2.3$ \\
Issa score & $2.9 \pm 0.9$ \\
Hypertension (\%) & 57.2 \\
Coronary artery disease (\%) & 25.2 \\
Acute myocardial infarction (\%) & 27.0 \\
Diabetes mellitus type 2 (\%) & 13.4 \\
Obesity (\%) & 9.1 \\
Number of medicines & $3.6 \pm 3.4$ \\
\hline
\end{tabular}

CAD, or AMI. We observed that SBP, DBP, and HR did not change significantly $(p>0.05)$ during 3 sequential measures in follow-up, regardless of pharmacotherapy for smoking cessation used and the presence of hypertension, $\mathrm{CAD}$, and/or previous AMI. The $\mathrm{CO}$ analysis showed a significant reduction $(p<0.001)$ in exhaled CO level in 3 sequential measures in follow-up in all the types of pharmacotherapy for smoking cessation used, alone or in combination.

We also analyzed BP and HR of followed patients $(n=900)$ according with a CO cutoff of $6 \mathrm{ppm}$ in the second and third visits and the presence of hypertension, CAD, or AMI. There was no significant influence of this variable in change $\mathrm{BP}$ and $\mathrm{HR}$ in all smoking cessation drugs group.

\section{Discussion}

It is well known that quitting smoking can reduce the risk of mortality from heart disease and acute myocardial infarction [2]. However, studies regarding CV risks associated with pharmacotherapies for smoking cessation have been concerned with public health. Few randomized controlled trials have been conducted in populations with high cardiovascular risk profiles and also few trials have compared head-to-head the safety of smoking cessation medications.

We did not observe any significant change in systolic or diastolic blood pressure and heart rate in 900 patients who used smoking cessation therapies and were followed at Heart Institute, a cardiac hospital with a large rate of patients with hypertension, CAD, and/or AMI. This result was regardless of the combination of pharmacotherapies used in the smoking cessation treatment and the presence or absence of cited comorbidities.

In the literature, tachycardia is a well-established and benign adverse effect observed in a lot of studies using NRT [5]. Some studies showed an increase in HR (1015 beats/min) and blood pressure $(5-10 \mathrm{mmHg}$ ) after NRT use $[19,20]$, even in smokers who interrupted tobacco consumption [20]. A recent meta-analysis showed similar results - an association of using NRT and increasing minor $\mathrm{CV}$ events, mainly due to the occurrence of tachycardia, but without adding risk to major $\mathrm{CV}$ events [6]. In patients with a history of a predisposing high-risk condition, in a smaller sample, this was not found [6]. The development of tolerance to blood pressure effects of nicotine in heavy smokers can be included in these divergent hemodynamic findings.

Concerning bupropion, an increase in blood pressure could be an important cardiovascular side effect, regardless of pre-existing hypertension, because of the effect of bupropion on the reuptake of norepinephrine [21]. However, this effect is not seen overall in clinical practice and our results are in accordance with the results from 
Table 2 Sequential measures of blood pressures, heart rate, and monoximetria of patients according to prescribed drugs and the presence of hypertension, CAD, or AMI

Varenicline
Variables
Initial SBP (mmHg)
Second visit SBP (mmHg)
Third visit SBP (mmHg)
p value
Initial DBP (mmHg)
Second visit DBP (mmHg)
Third visit DBP (mmHg)
$p$ value
Initial HR (bpm)
Second visit HR (bpm)
Third visit HR (bpm)
$p$ value
Initial monoximetria (ppm)
Second visit monoximetria (ppm)
Third visit monoximetria (ppm)
$p$ value

Presence of hypertension, $C A D$, or AMI

Varenicline Plus Bupropione

Presence of hypertension, CAD, or AMI

Variables
Initial SBP (mmHg)
Second visit SBP $(\mathrm{mmHg})$
Third visit SBP (mmHg)
$p$ value
Initial DBP (mmHg)
Second visit DBP (mmHg)
Third visit DBP (mmHg)
$p$ value
Initial HR (bpm)
Second visit HR (bpm)
Third visit HR (bpm)
$p$ value
Initial monoximetria (ppm)
Second visit monoximetria (ppm)
Third visit monoximetria (ppm)
$p$ value

$\begin{array}{cc}\text { No }(n=62) & \text { Yes }(n=49) \\ 117 \pm 14 & 128 \pm 18 \\ 117 \pm 15 & 126 \pm 21 \\ 115 \pm 15 & 129 \pm 21 \\ 0.56 & 0.63 \\ 75 \pm 8 & 81 \pm 12 \\ 74 \pm 8 & 81 \pm 12 \\ 74 \pm 8 & 81 \pm 11 \\ 0.85 & 0.27 \\ 79 \pm 10 & 73 \pm 10 \\ 74 \pm 13 & 73 \pm 10 \\ 77 \pm 10 & 73 \pm 11 \\ 0.06 & 0.86 \\ 17 \pm 10 & 16 \pm 8 \\ 11 \pm 10 & 10 \pm 7 \\ 7 \pm 7 & 9 \pm 8 \\ <0.001 & <0.001\end{array}$

Table 2 Sequential measures of blood pressures, heart rate, and monoximetria of patients according to prescribed drugs and the presence of hypertension, CAD, or AMI (Continued)

\begin{tabular}{lcc}
\hline Bupropione & & \\
& & \\
Variables & No $(n=39)$ & Yes $(n=42)$ \\
Initial SBP (mmHg) & $112 \pm 14$ & $132 \pm 27$ \\
Second visit SBP (mmHg) & $117 \pm 17$ & $126 \pm 23$ \\
Third visit SBP (mmHg) & $116 \pm 15$ & $132 \pm 28$ \\
$p$ value & 0.12 & 0.31 \\
Initial DBP (mmHg) & $74 \pm 7$ & $81 \pm 12$ \\
Second visit DBP (mmHg) & $76 \pm 10$ & $78 \pm 10$ \\
Third visit DBP (mmHg) & $76 \pm 9$ & $82 \pm 11$ \\
$p$ value & 0.63 & 0.14 \\
Initial HR (bpm) & $76 \pm 10$ & $73 \pm 13$ \\
Second visit HR (bpm) & $76 \pm 8$ & $73 \pm 14$ \\
Third visit HR (bpm) & $75 \pm 9$ & $74 \pm 14$ \\
$p$ value & 0.25 & 0.99 \\
Initial monoximetria (ppm) & $14 \pm 13$ & $12 \pm 11$ \\
Second visit monoximetria (ppm) & $7 \pm 10$ & $8 \pm 6$ \\
Third visit monoximetria (ppm) & $6 \pm 8$ & $7 \pm 6$ \\
$p$ value & $<0.001$ & $<0.001$
\end{tabular}

Bupropione Plus NRT

Variables

Initial SBP (mmHg)

Second visit SBP $(\mathrm{mmHg})$

Third visit SBP (mmHg)

$p$ value

Initial DBP (mmHg)

Second visit DBP $(\mathrm{mmHg})$

Third visit DBP $(\mathrm{mmHg})$

$p$ value

Initial HR (bpm)

Second visit HR (bpm)

Third visit HR (bpm)

$p$ value

Initial monoximetria (ppm)

Second visit monoximetria (ppm)

Third visit monoximetria (ppm)

$p$ value
Presence of hypertension, CAD, or AMI

$\begin{array}{cc}\text { No }(n=72) & \text { Yes }(n=211) \\ 117 \pm 16 & 132 \pm 21 \\ 117 \pm 18 & 134 \pm 22 \\ 118 \pm 18 & 132 \pm 21 \\ 0.89 & 0.53 \\ 73 \pm 10 & 80 \pm 12 \\ 73 \pm 11 & 79 \pm 13 \\ 74 \pm 11 & 80 \pm 13 \\ 0.67 & 0.81 \\ 76 \pm 11 & 73 \pm 14 \\ 75 \pm 13 & 74 \pm 12 \\ 75 \pm 11 & 74 \pm 13 \\ 0.87 & 0.83 \\ 11 \pm 7 & 11 \pm 8 \\ 7 \pm 6 & 7 \pm 8 \\ 5 \pm 6 & 5 \pm 5 \\ <0.001 & <0.001\end{array}$


Table 2 Sequential measures of blood pressures, heart rate, and monoximetria of patients according to prescribed drugs and the presence of hypertension, CAD, or AMI (Continued)

\begin{tabular}{lcc}
\hline NRT & & \\
& Presence of hypertension, CAD, or AMl \\
Variables & No $(n=62)$ & Yes $(n=209)$ \\
Initial SBP (mmHg) & $115 \pm 17$ & $130 \pm 23$ \\
Second visit SBP (mmHg) & $119 \pm 18$ & $128 \pm 23$ \\
Third visit SBP (mmHg) & $116 \pm 17$ & $130 \pm 22$ \\
p value & 0.48 & 0.65 \\
Initial DBP (mmHg) & $72 \pm 11$ & $78 \pm 14$ \\
Second visit DBP (mmHg) & $74 \pm 13$ & $77 \pm 13$ \\
Third visit DBP (mmHg) & $73 \pm 13$ & $77 \pm 13$ \\
$p$ value & 0.78 & 0.24 \\
Initial HR (bpm) & $75 \pm 11$ & $72 \pm 13$ \\
Second visit HR (bpm) & $74 \pm 10$ & $72 \pm 14$ \\
Third visit HR (bpm) & $72 \pm 10$ & $72 \pm 14$ \\
$p$ value & 0.10 & 0.90 \\
Initial monoximetria (ppm) & $10 \pm 7$ & $10 \pm 6$ \\
Second visit monoximetria (ppm) & $6 \pm 5$ & $5 \pm 5$ \\
Third visit monoximetria (ppm) & $4 \pm 4$ & $5 \pm 5$ \\
$p$ value & $<0.001$ & $<0.001$ \\
\hline
\end{tabular}

NRT nicotine replacement therapy (patch and/or gum), $C A D$ coronary artery disease, $A M I$ acute myocardial infarction, SBP systolic blood pressure, DBP diastolic blood pressure, $H R$ heart rate

other studies. Settle et al, enrolling more than 500 patients with depression, did not show clinically important effects on BP or HR when bupropion was compared with placebo [22]. In patients with $\mathrm{CV}$ diseases, a randomized trial including 248 smokers hospitalized because of CAD did not find a significant change in blood pressure after 12 weeks of slow release (SR) bupropion compared with placebo [7]. A clinical trial with 629 persistent smokers with CV disease, in which nearly half had experienced an AMI previously, evaluated bupropion versus placebo and did not find significant changes in BP or HR. Patients with baseline $\mathrm{BP} \geq 160 / 100 \mathrm{mmHg}$ were excluded [8]. In another study with 300 outpatient smokers with untreated stage 1 hypertension, different doses of bupropion induced a small reduction in BP, and bupropion $400 \mathrm{mg}$ SR also increased heart rate by 2.9 beats/min versus placebo [23]. On the other hand, studies by Roose et al with small samples observed that bupropion can increase sympathomimetic activity and increase $\mathrm{HR}$ and BP in patients with depression and heart disease if it is used in higher doses [24, 25].

In the same way, Tonstad et al reported no changes in BP after 24 weeks of varenicline versus placebo. The mean HR remained similar in the varenicline group and decreased by 2 beats per minute in the placebo group [26]. Additionally, a randomized trial with 714 smokers with stable CV disease (history of myocardial infarction, coronary revascularization, angina pectoris, peripheral arterial vascular disease, stroke or transient ischemic attack) showed a $0.5 \mathrm{mmHg}$ increase in SBP and no changes in DBP and HR in the varenicline group versus placebo [10].

There are some studies with the use of NRT in population of smokers without cardiovascular diseases or with hypertension alone $[27,28]$, which did not find changes in HR and BP.

This study has some limitations. BP and HR were measured at 3 consecutive visits. Therefore, although it is enough to have a comparison, possible small changes might be seen in a more prolonged follow. Compliance with the treatment and smoking status were measured during the follow-up period by asking patients and by measuring exhaled $\mathrm{CO}$ level, which showed a significant reduction in comparison with basal $\mathrm{CO}$ level, regardless of pharmacotherapy used. It indicates reduction or cessation of smoking. We did not design a study with the objective and power to observe major CV events.

Our findings of maintenance of BP and HR in a relatively large population of smokers with and without preexisting stable CV diseases using different combinations of smoking cessation therapies are important as a safety data for clinical practice. This result is in agreement with a recent meta-analysis of the use of NRT, bupropion and varenicline, which did not find an increase in major $\mathrm{CV}$ events with all drugs [6]. In patients with $\mathrm{CV}$ diseases, the existing data do not suggest harm and the benefits of smoking cessation in the long term exceeds an eventual small risk associated with pharmacotherapy drugs for smoking cessation.

\section{Conclusion}

Our data from a real clinical practice suggest that, even in patients with hypertension, CAD, and/or AMI, there are no significant clinical change in blood pressure or heart rate during the use of NRT, varenicline, and/or bupropion.

\begin{abstract}
Abbreviations
AMl: acute myocardial infarction; BP: blood pressure; CAD: coronary artery disease; CV: cardiovascular; DBP: diastolic blood pressure; HR: heart rate: MACE: Major Adverse Cardiovascular Event; NRT: nicotine replacement therapy; PAF: Program of Assistance to Smokers; SBP: systolic blood pressure.
\end{abstract}

\section{Competing interests}

Authors declare no competing interests.

\section{Authors' contributions}

JS conceived the study, and participated in its design and coordination and helped to draft the manuscript. APS participated in its design, data collect and drafting the manuscript. GGP, TOA and PVG participated of data collect date and helped to draft the manuscript. PCJLS and ACP participated in the design of the study and performed the statistical analysis. All authors read and approved the final manuscript. 


\section{Funding}

The authors declare this work was not supported.

\section{Author details}

${ }^{1}$ Heart Institute University of Sao Paulo Medical School, Sao Paulo, Brazil. ${ }^{2}$ Smoking Cessation Program Department, Heart Institute (InCor), University of Sao Paulo Medical School, Av. Dr. Eneas de Carvalho Aguiar 44, Cerqueira Cesar, 05403-900 Sao Paulo, SP, Brazil. 'Laboratory of Genetics and Molecular Cardiology, Heart Institute (InCor), University of Sao Paulo Medical School, Sao Paulo, Brazil.

\section{Received: 26 June 2015 Accepted: 21 December 2015}

Published online: 05 January 2016

\section{References}

1. Jha P, Ramasundarahettige C, Landsman V, Rostron B, Thun M, Anderson $\mathrm{RN}$, et al. 21st-century hazards of smoking and benefits of cessation in the United States. N Engl J Med. 2013;368:341-50. doi:10.1056/NEJMsa1211128.

2. Glantz S, Gonzalez M. Effective tobacco control is key to rapid progress in reduction of non-communicable diseases. Lancet. 2012:379:1269-71. doi:10.1016/S0140-6736(11)60615-6.

3. Fiore MC, Jaén CR, Baker TB, Bailey WC, Benowitz NL, Curry SJ, et al. Treating Tobacco Use and Dependence: 2008 Update.Clinical Practice Guideline. Rockville, MD: U.S. Department of Health and Human Services. Public Health Service. 2008.

4. DaCosta A, Guy JM, Tardy B, Gonthier R, Denis L, Lamaud M, et al. Myocardial infarction and nicotine patch: a contributing or causative factor? Eur Heart J. 1993;14:1709-11.

5. Mills EJ, Wu P, Lockhart I, Wilson K, Ebbert JO. Adverse events associated with nicotine replacement therapy (NRT) for smoking cessation. A systematic review and meta-analysis of one hundred and twenty studies involving 177,390 individuals. Tob Induc Dis. 2010; 8(1): 8. doi: 10.1186/16179625-8-8

6. Mills EJ, Thorlund K, Eapen S, Wu P, Prochaska JJ. Cardiovascular events associated with smoking cessation pharmacotherapies: a network metaanalysis. Circulation. 2014;129:28-41. doi:10.1161/CIRCULATIONAHA.113.003961.

7. Rigotti NA, Thorndike AN, Regan S, McKool K, Pasternak RC, Chang Y, et al. Bupropion for smokers hospitalized with acute cardiovascular disease. Am J Med. 2006;119:1080-7. doi:10.1016/j.amjmed.2006.04.024.

8. Tonstad S. Bupropion SR, for smoking cessation in smokers with cardiovascular disease: a multicentre, randomised study. Eur Heart J. 2003;24:946-55. doi:10.1016/50195-668X(03)00003-4.

9. Eisenberg MJ, Grandi SM, Gervais A, O'Loughlin J, Paradis G, Rinfret S, et al Bupropion for smoking cessation in patients hospitalized with acute myocardial infarction: a randomized, placebo-controlled trial. J Am Coll Cardiol. 2013;61:524-32. doi:10.1016/j.jacc.2012.08.1030.

10. Rigotti NA, Pipe AL, Benowitz NL, Arteaga C, Garza D, Tonstad S. Efficacy and safety of varenicline for smoking cessation in patients with cardiovascular disease: a randomized trial. Circulation. 2010;121:221-9. doi:10.1161/CIRCULATIONAHA.109.869008.

11. Singh S, Loke YK, Spangler JG, Furberg CD. Risk of serious adverse cardiovascular events associated with varenicline: a systematic review and meta-analysis. CMAJ. 2011;183:1359-66. doi:10.1503/cmaj.110218.

12. Prochaska JJ, Hilton JF. Risk of cardiovascular serious adverse events associated with varenicline use for tobacco cessation: systematic review and meta-analysis. BMJ. 2012;344, e2856. doi:10.1136/bmj.e2856

13. Issa JS, Abe TO, Moura S, Santos PCJL, Pereira AC. Effectiveness of coadministration of varenicline, bupropion, and serotonin reuptake inhibitors in a smoking cessation program in the real-life setting. Nicotine Tob Res. 2013;15(6):1146-50. doi:10.1093/ntr/nts230.

14. Ebbert JO, Hatsukami DK, Croghan IT, et al. Combination varenicline and bupropion SR for tobacco-dependence treatment in cigarette smokers. A randomized trial. JAMA. 2014;311(2):155-63. doi:10.1001/jama.2013.283185.

15. Rose JE, Behm FM. Combination varenicline/bupropion treatment in an adaptative smoking cessation paradigm. Am J Psychiatry. 2014;171(11): 1199-205. doi:10.1176/appi.ajp.2014.13050595.

16. Fagerström KO, Schneider NG. Measuring nicotine dependence: a review of the Fagerström Tolerance Questionnaire. J Behav Med. 1989;12:159-82.

17. Issa JS. A new nicotine dependence score and a new scale assessing patient comfort during smoking cessation treatment. J Bras Pneumol. 2012;38:761-5
18. Issa JS, Abe TM, Pereira AC, Megid MC, Shimabukuro CE, Valentin LS, et al. The effect of São Paulo's smoke-free legislation on carbon monoxide concentration in hospitality venues and their workers. Tob Control. 2011;20: 156-62. doi:10.1136/tc.2010.037614.

19. Najem B, Houssière A, Pathak A, Janssen C, Lemogoum D, Xhaët $O$, et al. Acute cardiovascular and sympathetic effects of nicotine replacement therapy. Hypertension. 2006;47:1162-7. doi:10.1161/01.HYP.0000219284. 47970.34.

20. Yugar-Toledo JC, Ferreira-Melo SE, Sabha M, Nogueira EA, Coelho OR, Consolin Colombo FM, et al. Blood Pressure circadian rhythm and endothelial function in heavy smokers: acute effects of transdermal nicotine. J Clin Hypertens (Greenwich). 2005;7:721-8.

21. Sobieraj DM, White WB, Baker WL. Cardiovascular effects of pharmacologic therapies for smoking cessation. J Am Soc Hypertens. 2013;7:61-7. doi:10.1016/j.jash.2012.11.003.

22. Settle EC, Stahl SM, Batey SR, Andrew Johnston J, Ascher JA. Safety profile of sustained-release bupropion in depression: Results of three clinical trials. Clin Ther. 1999;21:454-63. doi:10.1016/S0149-2918(00)88301-0.

23. Thase ME, Haight BR, Johnson MC, Hunt T, Krishen A, Fleck RJ, et al. A randomized, double-blind, placebo-controlled study of the effect of sustained-release bupropion on blood pressure in individuals with mild untreated hypertension. J Clin Psychopharmacol. 2008;28:302-7. doi:10.1097/JCP.0b013e318172424e

24. Roose SP. Considerations for the use of antidepressants in patients with cardiovascular disease. Am Heart J. 2000;140 Suppl 4:84-8.

25. Roose SP, Dalack GW, Glassman AH, Woodring S, Walsh BT, Giardina EG. Cardiovascular effects of bupropion in depressed patients with heart disease. Am J Psychiatry. 1991;148:512-6.

26. Tonstad S, Tønnesen P, Hajek P, et al. Effect of maintenance therapy with varenicline on smoking cessation. JAMA-EXPRESS. 2006;296:64-71.

27. Tanus-Santos JE, Carlos J, Toledo Y. Cardiovascular effects of transdermal nicotine in mildly hypertensive smokers. Am J Hypertens. 2001;14:610-4.

28. Zevin S, Jacob P, Benowitz NL. Dose-related cardiovascular and endocrine effects of transdermal nicotine. Clin Pharmacol Ther. 1998;64:87-95. doi:10.1016/S0009-9236(98)90026-1.

\section{Submit your next manuscript to BioMed Central and we will help you at every step:}

- We accept pre-submission inquiries

- Our selector tool helps you to find the most relevant journal

- We provide round the clock customer support

- Convenient online submission

- Thorough peer review

- Inclusion in PubMed and all major indexing services

- Maximum visibility for your research

Submit your manuscript at www.biomedcentral.com/submit
) Biomed Central 